\title{
Case Report \\ Neutropenic Enterocolitis Disclosing an Underlying Cyclic Neutropenia
}

\author{
Fatima Ezzahra Lahlimi (D), Khawla Khalil, Soumia Lahiaouni, and Illias Tazi \\ Hematology Department, University Hospital Mohamed VI, Faculty of Medicine, Cadi Ayyad University, Marrakech, Morocco \\ Correspondence should be addressed to Fatima Ezzahra Lahlimi; fatizahra02@hotmail.com
}

Received 10 July 2020; Revised 9 November 2020; Accepted 25 November 2020; Published 2 December 2020

Academic Editor: Juan Mejía-Aranguré

Copyright ( 92020 Fatima Ezzahra Lahlimi et al. This is an open access article distributed under the Creative Commons Attribution License, which permits unrestricted use, distribution, and reproduction in any medium, provided the original work is properly cited.

\begin{abstract}
Neutropenic enterocolitis is a syndrome characterized by fever and abdominal pain in a neutropenic patient. It is often reported in children treated for leukemia and rarely reported in patients with other diseases. Herein, we report the case of a 9-year-old patient with a medical history of recurrent fever and mouth ulcers since the age of 4, who presented with neutropenic enterocolitis complicated with intestinal perforation which all leaded to disclose cyclic neutropenia. The patient was successfully treated by aggressive supportive care combined with surgical intervention. Neutropenic enterocolitis with possible complications should be considered and promptly managed in every neutropenic patient and may reveal a rare cause of neutropenia as cyclic neutropenia.
\end{abstract}

\section{Introduction}

Neutropenic enterocolitis (NE), also named as typhlitis, is a critical condition that is associated with high mortality, due to inflammatory necrotization of the cecum, and it may extend to the ileum and ascending colon. It is mainly observed in patients profoundly myelosupressed [1-4].

Cyclic neutropenia $(\mathrm{CN})$ is a rare hematologic disorder that is characterized by repetitive episodes of neutropenia occurring at more or less regular intervals. During neutropenic periods, the commonest infections are pharyngitis, periodontis and cutaneous infections [5-7]. We report the case of cyclic neutropenia disclosed by neutropenic enterocolitis complicated with intestinal perforation. A brief overview of the relevant literature is given, focusing on the diagnosis and the optimal management of this rare lifethreatening association.

\section{Case Presentation}

A 9-year-old boy born to nonconsanguineous parents was admitted to the pediatric emergency department with complaints of progressive abdominal pain and fever during the last 24 hours. His medical history revealed recurrent episodes of fever, pharyngitis, and diarrhea since the age of 4 . The symptoms had cyclical pattern of 21 days and persisted for about a week. On the initial physical examination, he presented a fever of $39.6^{\circ} \mathrm{C}$, blood pressure at $90 / 60 \mathrm{mmhg}$, and heart rate at 100 beats per minute. There was an abdominal tenderness and guarding, especially in the right lower quadrant (RLQ). No superficial lymph nodes or hepatosplenomegaly were palpated. He had no pulmonary signs, and his heart examination was normal. The remainder of the examination was unremarkable.

Laboratory investigations on admission showed a total white blood cell count of $3000 / \mathrm{mm}^{3}$, an absolute neutrophil count of $340 / \mathrm{mm}^{3}\left(1500-8000 / \mathrm{mm}^{3}\right)$, a hemoglobin level of $13 \mathrm{~g} / \mathrm{dl}$, and a platelet count of $174000 / \mathrm{L}$. The bleeding time and coagulation time were normal. The C-reactive protein was $268 \mathrm{mg} / \mathrm{L}(<3 \mathrm{mg} / \mathrm{L})$. The lactate dehydrogenase $(\mathrm{LDH})$ was $420 \mathrm{UI} / \mathrm{L}(200-400)$.

A computed tomography (CT) scan of the abdomen showed signs of increased bowel wall thickness of the small intestines and ascending colon at $8 \mathrm{~mm}$ (normal $4 \mathrm{~mm}$ ). Bacterial and fungal cultures revealed no growth. Stool polymerase chain reaction assay was negative. Various other laboratory tests were performed. Serology for hepatitis A, hepatitis $B$, hepatitis $C$, human immunodeficiency virus, and 
cytomegalovirus was negative. Results of an Epstein-Barr virus serologic test were consistent with the previous exposure. Serologic tests were negative for antinuclear and antinative DNA antibodies. Bone marrow aspiration examination demonstrated a maturation arrest of granulocytic cells.

The patient was immediately started on ceftriaxone $100 \mathrm{mg} / \mathrm{kg} /$ day i.v. and amikacin $15 \mathrm{mg} / \mathrm{kg} /$ day i.v. according to the local epidemiology. He was switched to imipenem after 48 hours of persistent fever. He received IV morphine for the pain, fluid resuscitation, and correction of electrolyte imbalance. He was also put on bowel rest.

The patient's fever resolved two days later, and he experienced substantial improvement of abdominal pain. Neutrophil recovery was within 7 days, and repeated imaging after 2 weeks showed resolution of colonic inflammation and he was able to go back home.

Three weeks later, he was admitted to our emergency department for recurrence of fever and severe abdominal pain. He was neutropenic with an absolute neutrophil count at $340 / \mathrm{mm}^{3}$, and CT scan showed local necrosis, an intestinal perforation, and enterocutaneous fistulas (Figures 1 and 2). Urgent surgery was indicated. Laparotomy, resection of the involved bowel, and ileostomy were performed. Pathological analysis of the specimen revealed findings of diffuse loss of mucosa, hemorrhage, and a necrotic surface. The patient was immediately started on imipenem and received supportive measures (bowel rest with nasogastric decompression, fluid resuscitation, and supplemental nutrition).

Serial measurements of absolute neutrophil counts indeed demonstrated fluctuating neutrophil counts with a 21day periodicity and neutropenia at the nadir of the cycle, and a possibly underlying cyclic neutropenia was suspected. Additional tests (including detection of ELANE (elastase, neutrophil expressed) mutation) were not performed.

A granulocyte-colony-stimulating factor (G-CSF) treatment was started. Two days later, the patient's fever subsided. G-CSF therapy was continued at progressive doses till neutrophil recovery. The patient condition improved remarkably, and timely follow-up is being performed in order to manage further neutropenic episodes properly.

\section{Discussion}

Neutropenic enterocolitis (NE), also known as typhlitis, is an inflammatory process with intramural infection of the distal ileum, cecum, and ascending colon that occurs predominantly in neutropenic patients with leukemia. The clinical presentation is characterized with the triad of neutropenia, fever, and abdominal pain [1-4]. Its true occurrence is unknown. Its exact pathogenesis is poorly understood and believed to be multifactorial. The pathophysiological of cyclic neutropenia is incompletely defined. The majority of $\mathrm{CN}$ patients harbor ELANE (elastase, neutrophil expressed) mutations, but the precise mechanism of cyclic fluctuations in blood cells and the detailed molecular mechanisms are not understood. Premature apoptosis of myeloid precursors within the marrow is the cause of the reduced neutrophil production. It is due to the production of misfolded elastase

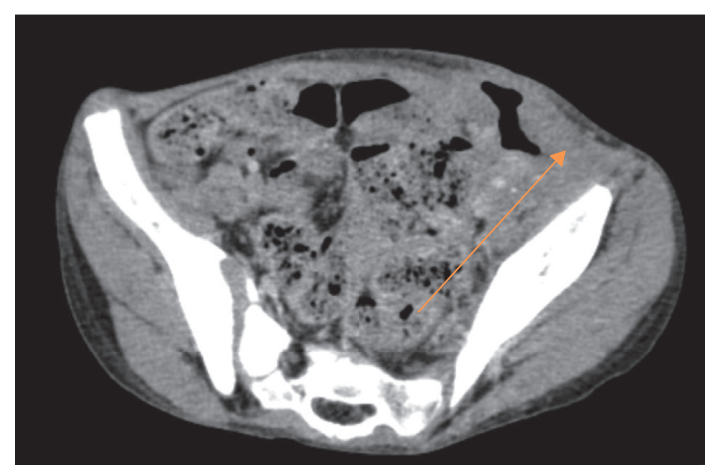

FIgURE 1: This collection is fistulized against the skin at the RLQ (arrow).

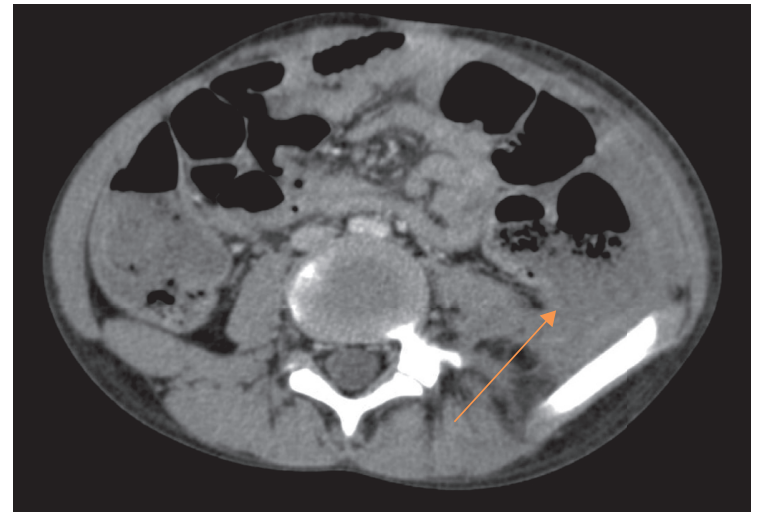

FIgURE 2: Abdominal CT: solution of continuity of the colonic wall with collection of neighbors (arrow).

proteins and may also induce the unfolded protein response $[8,9]$.

Neutropenia is the main risk factor as it causes impaired host defense to microbial invasion to the bowel wall. It is mainly described in leukemic patients who have received chemotherapy. It is also encountered in patients severely myelosuppressed due to therapy for solid tumors, autologous bone marrow transplant, and rarely in patients with cyclic neutropenia. Complications include necrosis, hemorrhage, perforation, and septicemia $[10,11]$.

When it comes to imaging characteristics, it is believed that CT is the imaging option of choice for the rapid diagnosis of NE with findings such as right lower quadrant inflammatory mass and pericecal fluid, inflammatory changes in the pericecal soft tissues, or bowel wall thickness of greater than $4 \mathrm{~mm}$. Abdominal X-ray may show small nonspecific signs such as bowel obstruction, a paucity of gas in the right lower quadrant, or a dilated and fluid-filled cecum. It can also show free intraperitoneal air, a complication of bowel perforation $[3,12]$.

Our patient presented initially with NE. CT scan showed signs of bowel wall thickness and was treated initially with antibiotics. However, the underlying cause remained a mystery.

Cyclic neutropenia $(\mathrm{CN})$ is a rare hematologic disease characterized by cyclic reduction in the granulocyte proliferative pool in the bone marrow and the release of mature 
neutrophils. It manifests typically at an approximately 21 day cycle with a range of 14 to 35 , lasting 3 to 6 days per episode. During the period when there are few circulating neutrophils, patients commonly suffer from fever, painful mouth ulcers, gingivitis, lymphadenopathy, pharyngitis, tonsillitis, and bacterial infections. Skin infections such as impetigo are also common. However, serious infections and septicemia are rare [5-10].

It is a very challenging diagnosis as the neutropenic phase only lasts for few days per cycle, and clinical manifestations such as recurrent fever and susceptibility to infection may be overlooked for a long time. Our patient spent the last five years with recurrent fever and mouth ulceration, and the diagnosis was only suspected after being admitted in our department.

It is because of the medical history of periodic fever and infection during the last 5 years, the admission for the NE, and the occurrence of fever, intestinal perforation, and neutropenia 21 days later that cyclic neutropenia was disclosed.

Early surgery is indicated in NE to treat complications such as perforation, peritonitis, gangrenous bowel, or severe gastrointestinal bleeding. Primary anastomosis is believed to have a high incidence of complications in neutropenic patients. Functioning ileostomy is recommended because of poor healing and risk of infection. Mortality rates range from 2.2 to $48 \%$, and the prognosis depends on the underlying disease and on the clinical conditions of the patient $[13,14]$.

\section{Conclusion}

Cyclic neutropenia can be easily overlooked due to its rarity and nonspecific symptoms. It may be the underlying cause to life-threatening conditions such as neutropenic enterocolitis and intestinal perforation.

\section{Abbreviations}

NE: Neutropenic enterocolitis

$\mathrm{CN}$ : Cyclic neutropenia

ELANE: Elastase, neutrophil expressed

G-CSF: Granulocyte-colony-stimulating factor

LDH: Lactate dehydrogenase

RLQ: Right lower quadrant.

\section{Data Availability}

No data were used to support this study.

\section{Conflicts of Interest}

The authors declare that they have no conflicts of interest.

\section{References}

[1] D. R. Urbach and O. D. Rotstein, "Typhlitis," CJS, vol. 42, no. 6, pp. 415-419, 1999.

[2] R. Xia and X. Zhang, "Neutropenic enterocolitis: a clinicopathological review," World Journal of Gastrointestinal Pathophysiology, vol. 10, no. 3, pp. 36-41, 2019.
[3] F. Mary, "Bavaro, neutropenic enterocolitis," Current Gastroenterology Reports, vol. 4, pp. 297-301, 2002.

[4] M. L. Davila, "Neutropenic enterocolitis," Current Opinion in Gastroenterology, vol. 22, no. 1, pp. 44-47, 2006.

[5] D. C. Dale, A. A. Bolyard, and A. Aprikyan, "Cyclic neutropenia," Seminars in Hematology, vol. 39, no. 2, pp. 89-94, 2002.

[6] T. Sotirova, "Cyclic neutropenia-case report," International Journal of Scientific Research, vol. 5, no. 2, pp. 2319-7064, 1983.

[7] M. Muhammad Sohail and K. Muhammad Bilal Salman, "A case of cyclic neutropenia in adults," JPMA, vol. 62, no. 3, 2012.

[8] I. Kollner, B. Sodeik, S. Schreek, H. Heyn, N. von Neuhoff, and M. Germeshausen, "Mutations in neutrophils elastase causing congenital neutropenia lead to cytoplasmic protein accumulation and induction of the unfolded protein response," Blood, vol. 108, pp. 493-500, 2006.

[9] D. S. Grenda, M. Murakami, J. Ghatak et al., "Mutations of the ELA2 gene found in patients with severe congenital neutropenia induce the unfolded protein response and cellular apoptosis," Blood, vol. 110, no. 13, pp. 4179-4187, 2007.

[10] A. Shafey, M. C. Ethier, J. Traubici, A. Naqvi, and L. Sung, "Incidence, risk factors, and outcomes of enteritis, typhlitis, and colitis in children with acute leukemia," Journal of Pediatric Hematology/Oncology, vol. 35, no. 7, pp. 514-517, 2013.

[11] L. V. Tyler, "Gastrointestinal complications in children with acute myeloid leukemia," Leukemia \& Lymphoma, vol. 51, no. 5, pp. 768-777, 2010.

[12] E. Altınel, N. Yarali, P. Isık, A. Bay, A. Kara, and B. Tunc, "Typhlitis in acute childhood leukemia," Medical Principles and Practice: International Journal of the Kuwait University, Health Science Centre, vol. 21, no. 1, pp. 36-39, 2012.

[13] N. Glavan, J. Roganović, L. Glavan-Gacanin, and N. Jonjic, "Appendectomy in a child with cyclic neutropenia in profound neutropenic episode," Therapeutics and Clinical Risk Management, vol. 11, pp. 1217-1219, 2015.

[14] N. Sundell, H. Boström, M. Edenholm, and J. Abrahamsson, "Management of neutropenic enterocolitis in children with cancer," Acta Paediatrica, vol. 101, no. 3, pp. 308-312, 2012. 